\title{
Investigation of Riemann Boundary Value Problem for Half Plane in Weighted Spaces of Analytic Functions
}

\author{
Zeynep Gokkus (Corresponding author) \\ Inebolu Vocational School, Computer Programming Program, \\ Kastamonu University, Kastamonu, Turkey \\ E-mail: zgokkus@gmail.com \\ Nizami Mustafa \\ Faculty of Science and Letters, Department of Mathematics, \\ Kafkas University, Kars, Turkey \\ E-mail: nizamimustafa@gmail.com
}

\begin{abstract}
The boundary value problems that deal with the piecewise continuous solution of an elliptic system that satisfies a certain jump condition on the curves for a given closed curve or a set of finite nonintersecting curves are Riemann boundary value problems.

In the first part of this study, the literature summary on the Riemann boundary value problem, the Riemann boundary value problem for the half plane and the Riemann boundary value problem for the half plane in the weighted spaces are given. In the second chapter, the Riemann boundary value problem for the half-plane in the weighted spaces is established, in the third chapter, Lemmas, which are the results obtained for the solution of the problem, are given together with their proofs, and finally, in the fourth chapter, two theorems and their proofs that indicate the necessary and sufficient conditions for the solution of the problem are given.
\end{abstract}

Keywords: Riemann boundary value problem, Riemann boundary value problem for half plane, Riemann boundary value problem for half plane in weighted spaces

DOI: $10.7176 / \mathrm{JSTR} / 7-01-06$

\section{Analitik Fonksiyonların A ğırlıklı Uzaylarda Yarı Düzlem İçin Riemann Sınır Değer Probleminin İncelenmesi}

\begin{abstract}
Özet
Belirli bir kapalı eğri veya sonlu kesişmeyen kapalı eğriler kümesi için, eğriler üzerinde belirli bir sıçrama koşulunu sağlayan bir eliptik sistemin parçalı sürekli çözümü ile ilgilenilen sınır değer problemleri Riemann sınır değer problemleridir.

Bu çalı̧̧manın birinci bölümünde Riemann sınır değer problemi, yarı düzlem için Riemann sınır değer problemi ve ağırlıklı uzaylarda yarı düzlem için Riemann sınır değer problemine ilişkin literatür özeti verilmiş̧ir. İkinci bölümde ağırlıklı uzaylarda yarı düzlem için Riemann sınır değer problemi kurulmuş, üçüncü bölümde problemin çözümüne ilişkin elde edilen sonuçlar niteliği taşıyan Lemmalar ispatlarılya birlikte verilmiş ve son olarak dördüncü bölümde problemin çözümü için gerek ve yeter koşulları belirten iki teoreme ve ispatlarına yer verilmiştir.
\end{abstract}

Anahtar kelimeler: Riemann sınır değer problemi, yarı düzlem için Riemann sınır değer problemi, ağırlıklı uzaylarda yarı düzlem için Riemann sınır değer problemi

\section{Giriş}

Sınır koşulları adı verilen koşullar altında kurulan bir diferansiyel denklemin çözümüne sınır değer problemi denilmektedir (Gakhov, 2014). On dokuzuncu yüzyılın sonlarına doğru, özellikle Rus bilim 
adamları tarafindan ilgi görmüş olan bu problemler günümüzde de çalışılmaya devam etmektedir (Vasilyev, 2018).

Belirli bir kapalı eğri veya sonlu kesişmeyen kapalı eğriler kümesi için, eğriler üzerinde belirli bir sıçrama koşulunu sağlayan bir eliptik sistemin parçalı sürekli çözümü ile ilgilenilen bu problem klasik sınır değer problemlerinden biridir ve Riemann sınır değer problemleri olarak anılmaktadır. Sınırlı bir bölgede tanımlı analitik fonksiyon problemleri ile ilgili ilk çalşsma Riemann'a (1857) dayandırılmaktadır (Brezis, 2003). Bu ilk çalışmada Hilbert (1904) Dirichlet probleminin çözümünü vermiştir. Konunun uygulamaları arasında lineer olmayan Poisson denklemleri, iki nokta sınır değer problemleri ve Laplace denklemlerinin çözümü yer almıştır (Görmüş, 2008).

Bu çalışmada analitik fonksiyonların yarı düzlem için Riemann sınır değer problemleri açıklanmıştır. İzleyen paragraflarda sırasıyla Riemann sınır değer problemleri ve yarı düzlem için Riemann sınır değer problemleri ile ilgili yapılan çalışmalar özetlenmiştir.

Gakhov (2014), ilk basım yılı 1966 olan kitabında sınır değer problemlerini, çeşitlerini, gerekli teoremleri ve ispatlarını, problemlerin çözüm ve uygulamalarına yer vererek açıklamıştır. Begehr ve Hile (1982), kuasilineer eliptik sistemler için doğrusal olmayan Riemann-Hilbert sınır değer problemini çözmek üzere Newton ve gömme yöntemlerinin bir kombinasyonu ile doğrusal olmayan problemleri doğrusal problemlere indirgemiştir. Du ve Wang (2005) polianalitik fonksiyonların Riemann sınır değer problemleri ve kapalı eğri üzerindeki metaanalitik fonksiyonları incelemiştir. Gürlebeck ve Zhang (2009) fonksiyonların bir $\mathrm{Cl}\left(V_{3,3}\right)$ Clifford cebrinde $\mathrm{Rm}(m>0)$ Riemann sinır değer problemlerini incelemiş ve büyüme davranıșı tahminlerini k-monojenik fonksiyonlar için seri açılımları ile birleştirerek harmonik ve biharmonik fonksiyonlar için genelleştirilmiş Liouville teoremini ispatlamıştır. Blaya vd. (2011), özellikle sınırdaki sıçrama davranışını göz önüne alarak, sınırın çok zayıf olduğu koşullarda Riemann sınır değer probleminin çözümü için düzeltilemeyen eğriler üzerinden eğrisel Cauchy integralini hesaplamıștır.

Alekna (1973) yarı düzlem için homojen Riemann sınır değer problemini incelemiştir. Nakhmein ve Nuller (1982), analitik fonksiyonlar teorisinin temel sınır problemleri olan, keyfi konturların eğik bir kafesi üzerindeki Riemann problemi, yarı düzlem için Hubert ve karma (mixed) problemlerini ve bir çizgi üzerinde periyodik yarıklar sistemi olan bir düzlem için Dirichlet problemini, translasyonel simetriye sahip belirli kontur sistemleri üzerinde ele alarak incelemiştir. Yine Nakhmein ve Nuller (1986), kısmen tam kaplinli ve kısmen kayma ile temas halinde olan bir damga (veya pul) ile temas halindeki elastik yarı düzlem problemlerini dikkate almıș, problemleri, birleşik Dirichlet-Riemann sınır değer problemine indirgemiş ve genel quadratik bir çözüm elde etmiştir. Xu ve Zhou (1993) çalışmalarında, üst yarı düzlemdeki monojenik fonksiyonlar için Riemann-Hilbert tipi sınır değer problemlerini hem klasik anlamda hem de $\mathrm{L}^{\mathrm{p}}$ anlamında incelemiş ve çözümlerin açık formüllerini elde etmiştir. Begehr ve Obolashvili (1994) bir Beltrami denkleminin yarı düzlem için Riemann sınır değer problemlerinin çözümünü vermiştir. Ming-hua (2006) analitik fonksiyonlar için ters Riemann-Hilbert sınır değer problemlerinin bir sınıfı için genel matematiksel formülleri vermiştir. Gaertner (2006) üst yarı düzlemde basit karmaşık sınır değer problemlerini incelemiştir. Sheng-yong (2011), yarı düzlemde analitik fonksiyonlar için ters Riemann-Hilbert sınır değer problemleri sınıfının matematiksel formülasyonunu vermiş, bu problemlerin klasik teorisine dayanarak, normal ve normal olmayan durumların ters sınır değer problemlerinin çözülebilirliğini tartışmış, ayrıca çözüm koşulları ve gösterimlerini vermiş̧ir. Galybin (2018) kitabında, gerilme ve yer değiştirme yönelimlerine göre ortaya çıkan elastik yarı düzlemler için sınır değer problemlerinin çözümlerinden bahsetmiştir. Hu vd. (2018), genelleştirilmiș $4 \times 4$ matrisli Ablowitz-Kaup-Newell-Segur-tipi Lax çifti ile izotropik bir ortamda polarize optik dalgaların yayılmasını tanımlayan, bütünleştirilebilir tutarlı bir şekilde bağlanmış doğrusal olmayan bir Schrödinger sistemini incelenmiş, karşıllık gelen başlangıç sınır değeri problemi, karmaşı düzlemde bir matris Riemann-Hilbert problemine indirgenmiş ve ayrıca ilişkili spektral fonksiyonların birbirine bağlı olduğunu ve global bir ilişki sağladığını göstermiștir. Hayrapetyan ve Aghekyan (2019a) çalışmalarında, ağırlıklı fonksiyonlar sınıfında yarı düzlem için Riemann sınır değer probleminin çözümüne ilişkin yöntemler incelemiştir. Yine Hayrapetyan ve Aghekyan (2019b), p(x) ağırlığına göre sürekli olan $C(p)$ fonksiyonlar sınıfında yarı düzlem için Riemann sınır değer problemini incelemiş, problemin çözümü için gerek ve yeter koşulları oluşturmuş ve çözümleri açık biçimde göstermiştir. Bikchantaev (2020), karakteristik denklem kökleri hakkında çeşitli varsayımlar altında yarı düzlemde sabit katsayılı ikinci dereceden doğrusal kısmi diferansiyel denklem için periyodik sınır değer problemlerini incelemiş, bu köklerin sanal kısımları aynı işarete sahipse Hilbert tipi bir sınır değer problemi ve zıt işaretlere sahiplerse Dirichlet tipi bir problemi düşünmüş, gerekli ve

66 | P a g e 
yeterli çözülebilirlik koşulları elde etmiş, bu problemlerin çözümü için açık formüller vermiş ve bunlara karşılık gelen homojen problemlerin çözüm sayısını elde etmiştir.

\section{Analitik Fonksiyonların Ağırlıkı Uzaylarda Yarı Düzlem İçin Riemann Sınır Değer Problemi}

$\Pi^{+}=\{x+i y, y>0\}$ ve $\Pi^{-}=\{x+i y, y<0\}$ sirasiyla üst ve alt yarı düzlemler olmak üzere A, $\Pi^{+} \cup \Pi^{-}$düzleminde analitik $\phi$ fonksiyonlarının bir sınıfı olsun ve aşağıdaki koşulları sağlasın.

$$
|\phi(z)| \leq C|z|^{m},|\operatorname{Imz}| \geq y_{0}>0
$$

Burada, m bir doğal sayıdır ve $C$ de muhtemelen $y_{0}$ 'a bağlı bir sabittir.

$f(+\infty)=f(-\infty) \neq 0$ olmak üzere $\bar{C}(-\infty ;+\infty)$, reel eksende sürekli olan $f(x)$ fonksiyonlarının $f(+\infty)$ ve $f(-\infty)$ ile sinırlı bir sinıfi olsun.

$\delta \in\left(\frac{1}{2}, 1\right]$ ve $x_{1}, x_{2}>C>0$ için $\bar{C}^{\delta}(-\infty ;+\infty), f \in \bar{C}(-\infty ;+\infty)$ 'i göstermek üzere aşağıdaki eşitsizlik yazılır

$$
\left|f\left(x_{1}\right)-f\left(x_{2}\right)\right| \leq M\left|\frac{1}{x_{1}}-\frac{1}{x_{2}}\right|^{\delta} .
$$

$\bar{C}(p)$ gerçel eksende sürekli f fonksiyonlarının bir sınıfıdır ve aşağıdaki formülle ifade edilmektedir.

$$
f(x) p(x) \in \bar{C}(-\infty ;+\infty) \text { ve }\|f\|_{\bar{C}(p)}=\max _{x \in(-\infty,+\infty)}|f(x) p(x)|
$$

Burada, $x_{k} \in(-\infty,+\infty)$ gerçel sayılar ve $\left\{a_{k}\right\}_{1}^{\infty}$ ve $\left\{x_{k}\right\}_{1}^{\infty}$ dizileri belirli koşulları sağlayan diziler olmak üzere, $p(x)$ aşağıdaki gibi gösterilmektedir

$$
p(x)=\prod_{k=1}^{\infty}\left|\frac{x-x_{k}}{x+i}\right|^{\alpha_{k}}, \quad 0<\alpha_{k}<1 .
$$

$\mathrm{Bu}$ aşamada Riemann sınır değer problemini yazabiliriz

$$
\phi^{+}(x)-a(x) \phi^{-}(x)=f(x) .
$$

Burada $\quad \Phi^{ \pm}(z), \quad L^{p}(p), p \in(1, \infty), p(x)=\prod_{k=1}^{N}\left|x_{k}-x\right|^{\alpha_{k}} \quad$ ağırlıklı uzaylarında tanımlı $\quad \Pi^{ \pm}$ bölgelerinde analitik olan fonksiyonları temsil etmektedir (Khvedelidze, 1956; Khevedelidze, 1970; Khevedelidze, 1975; Simonenko, 1959; Simonenko, 1964; Tovmasyan, 1994; Tovmasyan ve Babayan, 2007; Saldatov, 1991; Saldatov, 1993; Kazarian, 1987; Kazarian vd., 1997; Hatrapetyan, 2001; Hayrapetyan ve Babayan, 2001; Hayrapetyan ve Meliksetyan, 2003; Hayrapetyan, 2004).

Ağırlık fonksiyonu aşağıdaki biçimde olursa sınır değer problemi birim daire ile sınırlanmıştır

$$
p(t)=\prod_{k=1}^{\infty}\left|t_{k}-t\right|^{\alpha_{k}}, \quad 0<\alpha_{k}<1,\left|t_{k}\right|=1 .
$$

$\mathrm{Bu}$ problemde $\arg t_{k} \downarrow 0$ durumu $L^{1}(p)$ uzayında aşağıdaki eşitlik için araştırılmıştır (Hayrapetyan, 2018)

$$
\lim _{r \rightarrow 1-0}\left\|\varphi^{+}(r t)-a(t) \varphi^{-}\left(r^{-1} t\right)-f(t)\right\|_{L^{1}(p)}=0 .
$$

Burada, $\varphi^{-}(\infty)=0$ olmak üzere, $\varphi^{ \pm}(z)$ fonksiyonları, $D^{+}=\{z: z<1\}$ ve $D^{-}=\{z: z>1\}$ bölgelerinde analitiktir. Hayrapetyan (2018), $t_{k}, k=1,2, \ldots$ ve $\left(\alpha_{k}\right)_{1}^{\infty}$ koşulları için Riemann homojen probleminin $L^{1}(p)$ 'de sonsuz sayıda doğrusal bağımsız çözüme sahip olduğunu ve genel çözümü açık bir şekilde belirtmiştir. Ağırlık fonksiyonunun sınırda sonsuz sayıda sıfır değeri alması durumunda ağırlıklı uzaylar için Riemann sınır değer problemini araştırmak için önce $\phi \in A$ analitik fonksiyonu için $\alpha_{k}$ keyfi gerçel sayılar ve $x_{k}$ dizisi $x_{k} \in(-\infty,+\infty)$ olmak üzere, ağırlıklı uzaylar için Riemann sınır değeri problemini aşağıdaki gibi yazılmaktadır. 
Problem R: $f \in \bar{C}(p)$ olsun. Bu durumda $\Pi^{+} \cup \Pi^{-}$düzleminde analitik olan bir fonksiyon aşağıdaki gibi bulunur

$$
\lim _{y \rightarrow+0}\left\|\phi^{+}(x+i y)-a(x) \phi^{-}(x-i y)-f(x)\right\|_{\bar{C}(p)}=0
$$

Burada, $p(x)$ Eşitlik (1) olarak tanımlanan ifade, $a(x) \in \bar{C}^{\delta}(-\infty ;+\infty), a(x) \neq 0$ ve

$$
|x|>C>0 \text { 'da }|a(x)-a(\infty)|<C|x|^{-\delta} .
$$

Böylece, $a(x)$ katsayı indeksi 1'den büyük olduğunda homojen problemin doğrusal olarak bağımsız tek çözüme sahip olduğu ve aksi halde çözümü olmadığı gösterilmiştir (Hayrapetyan ve Aghekyan, 2019a). Ayrıca, homojen olmayan problemin çözümüne ulaşmak için gerekli koşulları da belirlenmiştir. Hayrapetyan ve Aghekyan (2019b)'in bu probleme ilişkin elde ettiği sonuçlar aşağıdaki gibi siralanabilir.

\section{Sonuçlar}

$\kappa=\operatorname{ind} a(t), t \in(-\infty,+\infty)$ olsun. $a_{1}(t)=\left(\frac{t+i}{t-i}\right)^{\kappa} a(t)$ ve ind $a_{1}(t)=0$ olmak üzere, (2) eşitlikleri aşağıdaki gibi yazılır

$$
\begin{gathered}
S^{+}(z)=\exp \left\{\frac{1}{2 \pi i} \int_{-\infty}^{+\infty} \frac{\ln a_{1}(t) d t}{t-z}\right\}, z \in \Pi^{+}, \\
S^{-}(z)=\left(\frac{z+i}{z-i}\right)^{\kappa} \exp \left\{\frac{1}{2 \pi i} \int_{-\infty}^{+\infty} \frac{\ln a_{1}(t) d t}{t-z}\right\}, z \in \Pi^{-} .
\end{gathered}
$$

Burada iki durum söz konusudur.

Durum 1: $\left\{x_{k}\right\}_{1}^{\infty}$ dizisi sonlu bir $x_{0}$ limitine sahip olduğunda,

$$
\sum_{k=1}^{\infty} \alpha_{k}<\infty
$$

Lemma 1. $\left\{x_{k}\right\}_{1}^{\infty}$ dizisi bazı c $>0$ sabitleri için aşă̆ıdaki koşulları să̆lasın.

$$
\begin{aligned}
& \sum_{k=1}^{\infty} \alpha_{k} \ln \left|x_{0}-x_{k}\right|>-\infty, \\
& \left|x_{k}-x_{j}\right|>c\left|x_{k}-x_{0}\right|, j \neq k .
\end{aligned}
$$

Bu aşamada aşă̆ıdaki eşitsizlik yazılır

$$
\text { inf } p_{m}=p_{0}>0, m=1,2, \ldots .
$$

Burada

$$
p_{m}=\prod_{k \neq m}^{\infty}\left|\frac{x_{m}-x_{k}}{x_{m}+i}\right|^{\alpha_{k}}
$$

İspat. Eşitlik (4) koşulunu kullanarak aşağıdaki ifadeler yazılır

$$
\begin{gathered}
\left|\frac{x_{j}-x_{k}}{x_{j}+i}\right|^{\alpha_{k}}>c^{\alpha_{k}}\left|\frac{x_{0}-x_{k}}{x_{j}+i}\right|^{\alpha_{k}}, \\
\prod_{k \neq j}^{\infty}\left|\frac{x_{j}-x_{k}}{x_{j}+i}\right|^{\alpha_{k}}>\prod_{k=1}^{\infty} c^{\alpha_{k}} \prod_{k \neq j}^{\infty}\left|\frac{x_{0}-x_{k}}{x_{j}+i}\right|^{\alpha_{k}} .
\end{gathered}
$$

Eşitlik (3) ile verilen koşula göre $\inf p_{m}=\delta>0, m=1,2, \ldots$ olacak şekilde bir $\delta>0$ vardır.

Durum 2: $\left\{x_{k}\right\}_{1}^{\infty}$ dizisinin limiti sonsuz olduğunda ise,

$$
\sum_{k=1}^{\infty} \alpha_{k} \ln \left|x_{k}\right|<\infty
$$


Lemma 2. $\left\{x_{k}\right\}_{1}^{\infty}$ dizisi bazı c $>0$ sabitleri için aşağıdaki koşulu sağlasın

$$
\left|x_{k}-x_{j}\right|>c x_{j}, j \neq k .
$$

Bu durumda,

$$
\inf p_{m}=p_{0}>0, m=1,2, \ldots .
$$

İspat. $c>0$ sabiti $\mathrm{k}$ ve j'den bağımsız olmak üzere, $\left|x_{k}-x_{j}\right|>c\left|x_{k}\right|, j \neq k$ için

$$
\prod_{k \neq j}^{\infty}\left|\frac{x_{j}-x_{k}}{x_{j}+i}\right|^{\alpha_{k}}>\prod_{k=1}^{\infty} c^{\alpha_{k}} \prod_{k \neq j}^{\infty}\left|\frac{x_{j}}{x_{j}+i}\right|^{\alpha_{k}}>C>0 .
$$

Böylece, inf $p_{m}=p_{0}>0, m=1,2, \ldots$ olduğu görülmektedir ve aşağıdaki eşitlikler yazılabilir

$$
\begin{gathered}
\delta_{k}(x)=\prod_{j \neq k}^{\infty}\left|\frac{x-x_{j}}{x+i}\right|^{\alpha_{j}} \mathrm{ve} \\
\delta_{x}=\delta_{k+1}(x)-\delta_{k}(x), \quad x \in\left[x_{k}, x_{k+1}\right) .
\end{gathered}
$$

Lemma 3. $\delta\left(x_{k}^{\prime}\right)=0$ olacak şekilde $x_{k}^{\prime} \in\left[x_{k}, x_{k+1}\right), k=1,2, \ldots$ vardır.

İspat.

$$
\begin{aligned}
\delta_{k+1}(x)-\delta_{k}(x) & =\prod_{j \neq k+1}^{\infty}\left|\frac{x_{j}-x}{x+i}\right|^{\alpha_{j}}-\prod_{j \neq k}^{\infty}\left|\frac{x_{j}-x}{x+i}\right|^{\alpha_{j}} \\
& =\left.\prod_{j \neq k, k+1}^{\infty}\left|\frac{x_{j}-x}{x+i}\right|^{\alpha_{j}} \cdot|| \frac{x_{k}-x}{x+i}\right|^{\alpha_{k}}-\left|\frac{x_{k+1}-x}{x+i}\right|^{\alpha_{k+1}} \mid .
\end{aligned}
$$

Burada, aşağıdaki ifadeleri sağlayacak biçimde $c_{1}, c_{2}>0$ seçilir

$$
\begin{gathered}
\delta\left(x_{k+1}-c_{1}\right)=\left|-c_{1}\right|^{\alpha_{k+1}}-\left|x_{k+1}-x_{k}-c_{1}\right|^{\alpha_{k}}<0 \mathrm{ve} \\
\delta\left(x_{k}+c_{2}\right)=\left|x_{k}-x_{k+1}-c_{2}\right|^{\alpha_{k}}-\left|c_{2}\right|^{\alpha_{k}}>0 .
\end{gathered}
$$

$\delta(x)$ sürekli olduğundan $\delta(x)=0$ denkleminin bir (tek) çözümü vardır. Bu noktalar $x_{k}^{\prime}$ ile gösterildiğinde Lemma 3 ispatlanmış olur.

$X_{1}=\left(-\infty, x_{1}^{\prime}\right)$ ve $X_{k}=\left[x_{k-1}^{\prime}, x_{1}^{\prime}, k=2,3, \ldots\right.$ olsun. Buradan, $X_{k} \cap X_{k+1}=\emptyset, k=1,2,3, \ldots$ olduğu açıktır.

Lemma 4. $\left\{x_{k}\right\}_{1}^{\infty}$ dizisi (3), (4) veya (5) koşullarından birini sağlasın. $O$ zaman $k=1,2, \ldots$ için aşăğdaki koşulu sağlayan bir $\delta>0$ vardır

$$
\inf f_{x \in X_{k}} \delta_{k}(x)>\delta>0 .
$$

İspat. $x \in\left(x_{k-1}^{\prime}, x_{k}\right)$ olsun. $\mathrm{O}$ zaman $j>k+1$ için $\left|x_{j}-x\right| \geq\left|x_{j}-x_{k}\right|$ ve eğer $j<k$ ise $\left|x_{j}-x\right|>$ $\left|x_{j}-x_{k-1}\right|$ olur. Lemma 1 ve 2 kullanılarak aşağıdaki ifadeler elde edilir

$$
\begin{gathered}
\prod_{j \geq k+1}\left|\frac{x_{j}-x}{x+i}\right|^{\alpha_{j}}>\prod_{j \geq k+1}\left|\frac{x_{j}-x_{k}}{x_{k}+i}\right|^{\alpha_{j}}>\delta>0 \mathrm{ve} \\
\prod_{j<k}\left|\frac{x_{j}-x}{x+i}\right|^{\alpha_{j}}>\prod_{j<k}\left|\frac{x_{j}-x_{k-1}}{x_{k}+i}\right|^{\alpha_{j}}>\delta .
\end{gathered}
$$

Dolayısıyla, $\delta_{k}(x) \geq \delta^{2}, x \in\left(x_{k-1}^{\prime}, x_{k}\right)$ olur.

Şimdi ise $x \in\left(x_{k}, x_{k}^{\prime}\right)$ olsun. O halde, $j<k$ iken $\left|x_{j}-x\right|>\left|x_{j}-x_{k}\right|$ ve 
Vol.7, No.1, 2021

$$
\prod_{j=1}^{k-1}\left|\frac{x_{j}-x}{x+i}\right|^{\alpha_{j}} \geq \prod_{j=1}^{k-1}\left|\frac{x_{j}-x_{k}}{x_{k+1}+i}\right|^{\alpha_{j}}>\delta
$$

olur. $j \geq k+1$ için ise $\left|x_{j}-x\right| \geq\left|x_{j}-x_{k+1}\right|$ ve

$$
\prod_{j>k}\left|\frac{x_{j}-x}{x+i}\right|^{\alpha_{j}}>\prod_{j>k}\left|\frac{x_{j}-x_{k+1}}{x_{k+1}+i}\right|^{\alpha_{j}}>\delta
$$

elde edilir ve dolayısıyla, $\delta_{k}>\delta^{2}$ olur.

Lemma 3 ve 4'e kullanılarak, $\tilde{\delta}(x)$ fonksiyonu süreklidir ve inf $\tilde{\delta}(x)>0, x \in(-\infty,+\infty)$ için aşağıdaki gibi gösterilir

$$
\tilde{\delta}(x)=\left\{\delta(x), x \in X_{k}\right\}, k=1,2, \ldots .
$$

$\phi(z)$ fonksiyonu ise aşağıdaki gibi tanımlanır

$$
\phi(z)=\frac{(z+i) S(z)}{2 \pi i} \int_{-\infty}^{+\infty} \frac{f(t)}{(t+i) S^{+}(t) \tilde{\delta}(t)} \frac{d t}{t-z}, z \in \Pi^{ \pm} .
$$

Lemma 5. C sabiti y'den bağımsız olduğunda aşağıdaki tahmin doğrudur

$$
\left\|\phi^{+}(x+i y)-a(x) \phi^{-}(x-i y)-f(x)\right\|_{\bar{C}(p)}=0 .
$$

Bu tahmin limit iliş̧kisi için de geçerlidir

$$
\lim _{y \rightarrow+0}\left\|\phi^{+}(x+i y)-a(x) \phi^{-}(x-i y)-f(x)\right\|_{\bar{C}(p)}=0 .
$$

İspat.

$$
\begin{aligned}
\phi^{+}(x+i y)-a(x) & \phi^{-}(x-i y) \\
= & \frac{(x+i y+i) S(z)}{2 \pi i} \int_{-\infty}^{+\infty} \frac{f(t)}{(t+i) S^{+}(t) \tilde{\delta}(t)} \frac{d t}{t-x-i y}- \\
& -\frac{a(x)(x-i y+i) S(x-i y)}{2 \pi i} \int_{-\infty}^{+\infty} \frac{f(t)}{(t+i) S^{+}(t) \tilde{\delta}(t)} \frac{d t}{t-x+i y} \\
& =I_{1}(f, x, y)+I_{2}(f, x, y) .
\end{aligned}
$$

Burada,

$$
I_{1}(f, x, y)=\frac{S(x+i y)(x+i y+i)}{\pi} \int_{-\infty}^{+\infty} \frac{f(t)}{(t+i) S^{+}(t) \tilde{\delta}(t)} \frac{y d t}{(t-x)^{2}+y^{2}}
$$

ve

$$
I_{2}(f, x, y)=\frac{1}{2 \pi i}(S(x+i y)(x+i y+i)-a(x) S(x-i y)(x-i y+i)) \cdot \int_{-\infty}^{+\infty} \frac{f(t)}{(t+i) S^{+}(t) \widetilde{\delta}(t)} \frac{d t}{t-x+i y} .
$$

Ayrica,

$$
I_{1}(f, x, y) p(x)=\frac{S(x+i y) p(x)}{\pi} \int_{-\infty}^{+\infty} \frac{f(t)(x+i y+i)}{(t+i) S^{+}(t) \tilde{\delta}(t)} \frac{y d t}{(t-x)^{2}+y^{2}} .
$$

$S^{+}(z)$ sınırlı ve $\tilde{\delta}(x)>\delta>0$ olduğundan (Lemma 4) aşağıdaki ifade edilir 


$$
\begin{aligned}
& \left|I_{1}(f, x, y) p(x)\right| \leq \widetilde{c_{1}} p(x) \int_{-\infty}^{+\infty}\left|\frac{f(t) y d t}{(t-x)^{2}+y^{2}}\right| \\
& +\widetilde{c_{1}} p(x) \int_{-\infty}^{+\infty}\left|\frac{f(t)((x+i y+i)-(t+i))}{t+i} \frac{y d t}{(t-x)^{2}+y^{2}}\right| \\
& =I_{1}^{(1)}(f, x, y)+I_{1}^{(2)}(f, x, y) .
\end{aligned}
$$

Dolayısiyla,

$$
I_{1}^{(1)}(f, x, y) \leq \widetilde{c_{1}} p(x) \max _{x \in(-\infty ;+\infty)}|f(x)| \cdot \int_{-\infty}^{+\infty} \frac{y|d t|}{(t-x)^{2}+y^{2}} \leq c_{1}\|f\|_{\bar{C}(p)} .
$$

$|(x+i y+i)-(t+i)|=|t-x-i y|$ için ise aşağıdaki ifade elde edilir

$$
\begin{aligned}
& I_{1}^{(2)}(f, x, y) \leq \widetilde{c_{2}} p(x) \max _{x \in(-\infty ;+\infty)}|f(x)| . \int_{-\infty}^{+\infty} \frac{y|d t|}{|t+i||t-x+i y|} \\
\leq & \widetilde{c_{2}}\|f\|_{\bar{C}(p)} \cdot\left\{\int_{-\infty}^{+\infty} \frac{y|d t|}{|t+i|^{2}}+\int_{-\infty}^{+\infty} \frac{y|d t|}{|t-x+i y|^{2}}\right\} \leq c_{2}\|f\|_{\bar{C}(p) .}
\end{aligned}
$$

Yukarıdaki ifadede $c_{1}, c_{2}$ sabitleri $y$ 'ye bağlı değildir. Bu nedenle $M_{1}, y^{\prime}$ ye bağlı bir sabit olmak üzere aşağıdaki bağıntı yazılabilir

$$
\left\|I_{1}(f, x, y)\right\|_{\bar{C}(p)} \leq M_{1}\|f\|_{\bar{C}(p)}
$$

Ayrica,

$I_{2}(f, x, y) p(x)=\frac{p(x)}{2 \pi i}(S(x+i y)(x+i y+i)-a(x) S(x-i y)(x-i y+i)) \int_{-\infty}^{+\infty} \frac{f(t)}{(t+i) S^{+}(t) \tilde{\delta}(t)} \frac{d t}{t-x+i y}$.

$S^{+}$ve $S^{-}$ile sınırlı olan aşağıdaki ifade gibi

$$
\begin{gathered}
S^{+}(x+i y)(x+i y+i)-a(x) S^{-}(x-i y)(x-i y+i)=(x+i)\left(S^{+}(x+i y)-a(x) S^{-}(x-i y)\right)+ \\
i y\left(S^{+}(x+i y)+a(x) S^{-}(x-i y)\right) .
\end{gathered}
$$

Lemma 1 (Aghekyan, 2016) kullanılarak $y>0$ ve bir $A>0$ sabiti için aşağıdaki tahmin elde edilir

$$
\left|S^{+}(x+i y)-a(x) S^{-}(x-i y)\right|<A \frac{y^{\delta}}{|x+i|^{2 \delta}}
$$

Buradan, $M_{2}, y^{\prime}$ den bağımsız bir sabit olmak üzere,

$$
I_{2}(f, x, y) \leq \widetilde{M_{2}} p(x) \max _{x \in(-\infty ;+\infty)}|f(x)| \int_{-\infty}^{+\infty} \frac{|d t|}{|t+i||t-x+i y|} \leq M_{2}\|f\|_{\bar{C}(p)}
$$

yazılır. Dolayısıyla, $M=\max \left\{M_{1}, M_{2}\right\}$ olmak üzere,

$$
\left\|\phi^{+}(x+i y)-a(x) \phi^{-}(x-i y)\right\|_{\bar{C}(p)} \leq M\|f\|_{\bar{C}(p)} .
$$

Lemma 5 'in ikinci kısmının ispatı için, $f_{n}(x) \in \bar{C}^{\delta}(p)$ aşağıdaki koşulu sağlayan bir sonlu fonksiyonlar dizisi olsun

$$
\lim _{n \rightarrow \infty}\left\|f_{n}(x)-f(x)\right\|_{\bar{C}(p)}=0
$$

Keyfi bir $n$ için 
Vol.7, No.1, 2021

$$
\phi_{n}(z)=\frac{(z+i) S(z)}{2 \pi i} \int_{-\infty}^{+\infty} \frac{f_{n}(t)}{(t+i) S^{+}(t) \tilde{\delta}(t)} \frac{d t}{t-z}, \quad z \in \Pi^{ \pm}
$$

Bu aşamada (7)'nin sağlandığı gösterilmelidir.

$$
\lim _{y \rightarrow+0}\left\|\phi_{n}^{+}(x+i y)-a(x) \phi_{n}^{-}(x-i y)-f_{n}(x)\right\|_{\bar{C}(p)}=0 .
$$

Sokhotski-Plemelj formülünden (8) elde edilir

$$
\phi_{n}^{+}(x+i y)-a(x) \phi_{n}^{-}(x-i y)=f_{n}(x), x \in(-A, A) .
$$

Eğe,$|x|>A$ ise

$$
\begin{array}{r}
J_{1}(x, y)=\frac{S^{+}(x+i y)(x+i y+i)}{2 \pi i} \int_{-\infty}^{+\infty} \frac{f_{n}(t)}{(t+i) S^{+}(t) \tilde{\delta}(t)} \frac{y d t}{(t-x)^{2}+y^{2}}, \\
J_{2}(x, y)=\frac{1}{2 \pi i}(S(x+i y)(x+i y+i) \\
\quad-a(x) S(x-i y)(x-i y+i)) \cdot \int_{-\infty}^{+\infty} \frac{f(t)}{(t+i) S^{+}(t) \tilde{\delta}(t)} \frac{y d t}{t-x+i y}
\end{array}
$$

olmak üzere, aşağıdaki gösterim kullanılır

$$
\phi_{n}^{+}(x+i y)-a(x) \phi_{n}^{-}(x-i y)=J_{1}(x, y)+J_{2}(x, y) .
$$

Buradan da

$$
\begin{aligned}
& \max _{|z|>a} p(x)\left|\phi_{n}^{+}(x+i y)-a(x) \phi_{n}^{-}(x-i y)-f_{n}(x)\right| \\
& \quad \leq \max _{|z|>a} p(x)\left|J_{1}(x, y)\right|+\max _{|z|>a} p(x)\left|J_{2}(x, y)\right|
\end{aligned}
$$

elde edilir.

$J_{1}(x, y)$ için $f_{n}(x)=0,|x|>A$ ve $\mathrm{C}$ sabit olduğundan aşağıdaki tahminin yapılması mümkündür

$$
\begin{gathered}
p(x) J_{1}(x, y) \leq C \max _{|z|>a}\left\{p(x) y|x+i y+i| \int_{-\infty}^{+\infty}\left|\frac{f_{n}(t)}{(t+i) S^{+}(t)}\right| \frac{y d t}{(t-x)^{2}+y^{2}}\right\} \\
\leq C\left\|f_{n}(x)\right\|_{\bar{C}(p)} \max _{|z|>a}\left\{y|x+i y+i| \int_{-2 A}^{+2 A}\left|\frac{f_{n}(t)}{(t+i) S^{+}(t)}\right| \frac{d t}{(t-x)^{2}}\right\} \\
=C_{1}\left\|f_{n}(x)\right\|_{\bar{C}(p)} \max _{|z|>a}\left\{\frac{y|x+i y+i|}{\left|x^{2}-4 A^{2}\right|}\right\} .
\end{gathered}
$$

Böylece, $y \rightarrow+0$ iken $J_{1}(x, y)=0$ eğilimindedir.

Lemma 5'in ilk kısmının ispatında benzer şekilde $y \rightarrow+0$ iken $J_{2}(x, y)=0$ eğiliminde olduğu görülebilir. Dolayısıyla, Eşitlik (8) kullanılarak (7) elde edilir.

Lemma 5'in ilk tahmini ve (7) kullanılarak aşağıdaki ikinci tahmin elde edilir 


$$
\begin{aligned}
\| \phi^{+}(x+i y)-a & (x) \phi^{-}(x-i y)-f(x) \|_{\bar{C}(p)} \\
& \leq\left\|\phi_{n}^{+}(x+i y)-a(x) \phi_{n}^{-}(x-i y)-f_{n}(x)\right\|_{\bar{C}(p)}+\left\|f_{n}(x)-f(x)\right\|_{\bar{C}(p)} \\
& +\left\|\phi_{n}^{+}(x+i y)-\phi^{+}(x+i y)-a(x)\left[\phi_{n}^{-}(x-i y)\right]-\phi^{-}(x-i y)\right\|_{\bar{C}(p)} \\
& \leq\left\|\phi_{n}^{+}(x+i y)-a(x) \phi_{n}^{-}(x-i y)-f_{n}(x)\right\|_{\bar{C}(p)}+2\left\|f_{n}(x)-f(x)\right\|_{\bar{C}(p)} .
\end{aligned}
$$

Dolayısiyla,

$$
\lim _{y \rightarrow+0}\left\|\phi_{n}^{+}(x+i y)-a(x) \phi_{n}^{-}(x-i y)-f_{n}(x)\right\|_{\bar{C}(p)}=0 .
$$

Hayrapetyan ve Aghekyan (2019b) yukarıda verilen sonuçların yer aldığı iki teoremi ve ispatlarını aşağıdaki gibi vermişlerdir.

\section{Temel Teoremler}

Teorem 1. (9) problem düşünülsün.

$$
\lim _{y \rightarrow+0}\left\|\phi_{n}^{+}(x+i y)-a(x) \phi_{n}^{-}(x-i y)\right\|_{\bar{C}(p)}=0 .
$$

a) $\quad \kappa \geq 0$ olduğunda $A, B$ sabitleri ve $S(z)$ de (2) ile tanımlı eşitliklerin birleşimini göstermek üzere (9) probleminin genel çözümü $(A z+B) S(z)$ 'dir.

b) $\quad \kappa=-1$ iken (9) probleminin genel çözümü $A(z+i) S(z)^{\prime}$ dir.

c) Diğer durumlarda (9) homojen probleminin çözümü yoktur.

İspat. $x_{0},\left\{x_{k}\right\}_{1}^{\infty}$ noktalarının toplamı olan bir nokta ve $P(z)$ de $\mathrm{m}$ dereceli bir polinom olmak üzere, (9) probleminin çözümü ya

$$
P(z) S(z), \frac{S(z)}{x_{k}-z}, k=1,2 \text { veya } \frac{S(z)}{x_{0}-z} .
$$

(6) kullanılarak,

$$
\begin{aligned}
\mid S^{+}(x+i y)(x+ & i y)^{k}-a(x) S^{-}(x-i y)(x-i y)^{k} \mid \\
& \leq\left|(x+i y)^{k}\right|\left|S^{+}(x+i y)-a(x) S^{-}(x-i y)\right| \\
& +\left|(x+i y)^{k}\right|\left|a(x) S^{-}(x-i y)\right| \cdot\left|1-\left(\frac{x-i y}{x+i y}\right)^{k}\right| \\
& \leq\left|(x+i y)^{k}\right|\left(\left|S^{+}(x+i y)-a(x) S^{-}(x-i y)\right|+\frac{A_{1} y}{|x|}\right) \leq y\left(A|x|^{k-1}+y^{\delta}|x|^{k-2 \delta}\right)
\end{aligned}
$$

elde edilir.

Buradan, eğer $\kappa \geq 0$ ise

$$
\left|P(x+i y) S^{+}(x+i y)-a(x) P(x-i y) S^{-}(x-i y)\right| \leq y\left(A|x|^{m-1}+y^{\delta}|x|^{m-2 \delta}\right) .
$$

Dolayısıyla, (9) probleminin bir çözümünün $P(z) S(z)$ olması için $m$ değeri 0 veya 1 olmalıdır.

Böylece, çözüm $(A z+B) S(z)$ ile temsil edilir.

Eğer $\kappa=-1$ ise $S^{-}(z), z=-i$ noktasinda 1. dereceden kutba sahiptir. Bu nedenle $P(z), A(z+i) S(z)$ biçiminde gösterilir.

$\kappa<-1$ olması durumunda, $z=-i$ noktasında $|\kappa|$. dereceden kutba sahiptir. Çünkü $m \leq 1$ için (9) probleminin çözümü yoktur.

Bu aşamada $\frac{S(z)}{x_{k}-z}$ ve $\frac{S(z)}{x_{0}-z}$ 'nin (9) probleminin koşulunu sağlamadığını göstermek gerekmektedir.

Koşulun sağlandığı varsayıldığında 


$$
\phi_{1}(z)=\frac{S(z)}{x_{k}-z}
$$

yazılır ve

$$
\phi_{1}^{+}(x+i y)-a(x) \phi_{1}^{-}(x-i y)=\frac{S(x+i y)}{x_{k}-x-i y}-a(x) \frac{S(x-i y)}{x_{k}-x+i y}=I_{1}(x, y)+I_{2}(x, y) .
$$

Burada,

$$
I_{1}(x, y)=\frac{2 i y S(x+i y)}{\left(x_{k}-x\right)^{2}+y^{2}} \text { ve } I_{2}(x, y)=\frac{S(x+i y)+a(x) S(x-i y)}{x_{k}-x+i y} .
$$

$x=x_{k}$ olmas 1 durumunda

$$
\left|I_{1}(x, y) p(x)\right|=C \frac{p(x)}{y}
$$

elde edilir. Dolayısıyla,

$$
\lim _{y \rightarrow+0}\left\|I_{1}(x, y)\right\|_{\bar{C}_{p}}=\infty .
$$

Şimdi ise (6) eşitsizliği kullanılarak $y \rightarrow+0$ 'a benzer şekilde aşağıdaki ifade elde edilir

$$
\left|I_{2}(x, y) \cdot p(x)\right| \leq \widetilde{c_{2}} \frac{y^{\delta}}{|x+i|^{2 \delta}} \frac{\left|x-x_{k}\right|^{\alpha^{k}}}{\left|x_{k}-x+i y\right|} \leq c_{2} \frac{y^{\delta}}{|x+i|^{2 \delta}} \frac{\left|x-x_{k}\right|^{\alpha^{k}}}{\left|x_{k}-x\right|^{\frac{1}{2}} y^{\frac{1}{2}}}=c_{2} \frac{y^{\delta-\frac{1}{2}}}{\left|x-x_{k}\right|^{\frac{1}{2}-\alpha_{k}}} \rightarrow 0 .
$$

Böylece,

$$
\lim _{y \rightarrow+0}\left\|I_{2}(x, y)\right\|_{\bar{C}_{p}}=\infty
$$

Bu nedenle $\phi_{1}^{+}(z),(9)$ probleminin bir çözümü değildir.

Ayrica,

$$
\phi_{0}(z)=\frac{S(z)}{x_{0}-z}
$$

eşitliği ile (9) probleminin koşulunun sağlandığı varsayılarak da benzer bir sonuç aşağıdaki gibi elde edilir

$$
\phi_{0}^{+}(x+i y)-a(x) \phi_{0}^{-}(x-i y)=J_{1}(x, y)+J_{2}(x, y)
$$

Burada,

$$
J_{1}(x, y)=\frac{2 i y S(x+i y)}{\left(x_{0}-x\right)^{2}+y^{2}} \text { ve } J_{2}(x, y)=\frac{S(x+i y)+a(x) S(x-i y)}{x_{0}-x+i y}
$$

$k \rightarrow \infty$ gibi $x_{k} \rightarrow x_{0}$ olduğu dikkate alındığında,

$$
\lim _{y \rightarrow+0}\left\|J_{1}(x, y)\right\|_{\bar{C}_{p}}=\infty \text { ve } \lim _{y \rightarrow+0}\left\|J_{2}(x, y)\right\|_{\bar{C}_{p}}=0
$$

elde edilir. Sonuç olarak $\phi_{0}(z)$ de (9) probleminin bir çözümü değildir.

Teorem 2. $f \in \bar{C}_{p}$ olsun ve $\left\{x_{k}\right\}_{1}^{\infty}$ noktalar dizisi (3), (4) veya (5) koşullarından birini să̆lasın. Bu durumda, aşă̆ıdaki önermeler doğrudur.

a) Ĕger $\kappa \geq 0$ ise $z \in \Pi^{ \pm}$ve A, B sabitler olmak üzere homojen olmayan Problem R'nin genel çözümü aşă̆ldaki gibidir 


$$
\phi(z)=\frac{(z+i) S(z)}{2 \pi i} \int_{-\infty}^{+\infty} \frac{f(t)}{(t+i) S^{+}(t) \tilde{\delta}(t)} \frac{d t}{t-z}+(A z+B) S(z) .
$$

b) Ĕğer $\kappa=-1$ ise homojen olmayan Problem R'nin genel çözümü aşağıdaki gibi gösterilebilir

$$
\phi(z)=\frac{(z+i) S(z)}{2 \pi i} \int_{-\infty}^{+\infty} \frac{f(t)}{(t+i) S^{+}(t) \tilde{\delta}(t)} \frac{d t}{t-z}+(z+i) S(z), z \in \Pi^{ \pm} .
$$

c) Ĕger $\kappa<-1$ ise homojen olmayan Problem R'nin çözülebilmesi için gerek ve yeter şart $f$ fonksiyonunun aşağıdaki koşulları sağlamasıdır

$$
\int_{-\infty}^{+\infty} \frac{f(t)}{S^{+}(t) \widetilde{\delta}(t)} \frac{d t}{(t+i)^{j}}=0, \quad j=1,2, \ldots,-\kappa-1 .
$$

Bu durumda genel çözüm, $A=B=0$ olmak üzere (11) ile gösterilebilir.

İspat. a) ve b) şıklarındaki önermeler Lemma 4 ve Teorem 1 ile ispatlanabilir.

c) $\kappa<-1$ olsun. Bu durumda,

$$
\phi^{+}(x+i y)-a(x) \phi^{-}(x-i y)=f_{y}(x) .
$$

Eşitlik (12), $(x+i)^{-1}$ ile çarpılır ve $a_{x}=\frac{s^{+}(x)}{s^{-}(x)}$ olduğu dikkate alınırsa,

$$
\frac{\phi^{+}(x+i y)}{S^{+}(x)(x+i)}-\frac{\phi^{-}(x-i y)}{S^{-}(x)(x+i)}=\frac{f_{y}(x)}{S^{+}(x)(x+i)} .
$$

Buradan da,

$$
\phi_{y}^{+}(z)=\frac{\phi^{+}(z+i y)}{S^{+}(z)(z+i)}, \quad z \in \Pi^{+} \text {ve } \phi_{y}^{-}(z)=\frac{\phi^{-}(z-i y)}{S^{-}(z)(z+i)}, \quad z \in \Pi^{-}
$$

yazılır ve bu ifadelerin arasındaki fark aşağıdaki gibi elde edilir

$$
\phi_{y}^{+}(x)-\phi_{y}^{-}(x)=\frac{f_{y}(x)}{S^{+}(x)(x+i)} .
$$

$\kappa<-1$ olması durumunda, $\phi_{y}^{-}(z)$ fonksiyonu $z=-i$ noktasında $|\kappa-1|$ mertebeden sıfira sahiptir.

Sonuç olarak $f(x)$ fonksiyonu aşağıdaki koşulları sağlar

$$
\int_{-\infty}^{+\infty} \frac{f(t)}{S^{+}(t) \tilde{\delta}(t)} \frac{d t}{(t+i)^{j}}=0, \quad j=1,2, \ldots,-\kappa-1 .
$$

\section{Kaynakça}

Riemann, B. (1902). Bernhard Riemann's gesammelte mathematische Werke: Nachträge. BG Teubner.

Hilbert, D. (1904). Über das Dirichletsche Prinzip. Mathematische Annalen, 59(1-2), 161-186.

Khvedelidze, B. V. (1956). On discontinuous Riemann-Privalov problem for several functions. Soobshch. AN Gruz. SSR, 17, 865-872.

Simonenko, I. B. (1959). RIEMANN BOUNDARY VALUE PROBLEM WITH A CONTINUOUS COEFFICIENT. DOKLADY AKADEMII NAUK SSSR, 124(2), 278-281.

Simonenko, I. B. (1964). The Riemann boundary-value problem for $\mathrm{n}$ pairs of functions with measurable coefficients and its application to the study of singular integrals in L_p spaces with weights. Izvestiya Rossiiskoi Akademii Nauk. Seriya Matematicheskaya, 28(2), 277-306.

Khvedelidze, B. (1970). On singular integral operator and Cauchy type integral. Reports of National Academy of Georgia SSSR, 28, 73-84. 
Alekna, P. Y. (1973). On a homogeneous Riemann boundary-value problem with an infinite index of logarithmic order for a halfplane. Mathematical transactions of the Academy of Sciences of the Lithuanian SSR, 13(3), 349-355.

Khvedelidze, B. V. (1975). The method of Cauchy type integrals in discontinuous boundary value problems of the theory of holomorphic functions of a complex variable. Itogi Nauki i Tekhniki. Seriya" Sovremennye Problemy Matematiki. Noveishie Dostizheniya", 7, 5-162.

Begehr, H., and Hile, G. N. (1982). Nonlinear Riemann boundary value problems for a nonliear elliptic system in the plane. Mathematische Zeitschrift, 179(2), 241-261.

Nakhmein, E. L., and Nuller, B. M. (1982). On quasiperiodic boundary value problems and their applications in the theory of elasticity. Journal of Applied Mathematics and Mechanics, 46(5), 658-666

Nakhmein, E. L., and Nuller, B. M. (1986). Contact between an elastic half-plane and a partly separated stamp. Journal of Applied Mathematics and Mechanics, 50(4), 507-515.

Kazarian, K. C. (1987). Summability of generalized Fourier series and Dirichlet's problem inL p $(\mathrm{d} \mu)$ and weightedH p-spaces (p> 1). Analysis Mathematica, 13(3), 173-197.

Soldatov, A. P. (1991). A function theory method in boundary value problems in the plane. I. The smooth case. Izvestiya Rossiiskoi Akademii Nauk. Seriya Matematicheskaya, 55(5), 1070-1100.

Soldatov, A. P. (1993). A Function Theory Method in Elliptic Problems in the Plane. II. The Piecewise Smooth Case. Russian Academy of Sciences. Izvestiya Mathematics, 40(3), 529.

Xu, Z., and Zhou, C. (1993). On boundary value problems of riemann-hilbert type for monogenic functions in a half space of. Complex Variables and Elliptic Equations, 22(3-4), 181-193.

Begehr, H., and Obolashvili, E. (1994). Some boundary value problems for a Beltrami equation. Complex Variables and Elliptic Equations, 26(1-2), 113-122.

Tovmasyan, N. E., Zakaryan, G. V., and Gevorkyan, L. Z. (1994). Boundary value problems for partial differential equations and applications in electrodynamics. World Scientific.

Kazarian K., Soria F., and Spitkovsky I. (1997)., The Riemann boundary value problem in spaces with weight admitting singularities, Dokl., 357, no. 6, pp. 717-719.

Hayrapetyan, H. (2001). On the Dirichlet problem in spaces with weight in a halfplane. Proceedings of NAS of Armenia. Mathematics, 36(6), 7-15.

Hayrapetyan, H. and Babayan V. (2001). On the Dirichlet problem in the space of continuous functions with weight (in Russian), Belgorod State University Scienti_c Bulletin, Series: Mathematics. Physics, 112, no. 17, pp. 5-16.

Hayrapetyan, H. and Meliksetyan, P. (2003). The Hilbert boundary value problem in a halfplane in spaces with weight (in Russian), Proceedings of the NAS of Armenia. Mathematics, 38, no. 6, pp. 17-32.

Brezis, H. (2003). The interplay between analysis and topology in some nonlinear PDE problems. Bulletin of the American Mathematical Society, 40(2), 179-201.

Hayrapetyan, H. (2004). Dirichlet problem in the half-plane for RO-varying weight functions. Topics in Analysis and its Aplications. Dordrecht/Boston/London. Kluwer Academic Publishers, 147, 311-317.

76 | P a g e

www.iiste.org 
Du, J., and Wang, Y. (2005). Riemann boundary value problems of polyanalytic functions and metaanalytic functions on the closed curves. Complex Variables, Theory and Application: An International Journal, 50(7-11), 521-533.

Ming-hua, W. A. N. G. (2006). A class of inverse Riemann-Hilbert boundary value problems [J]. Pure and Applied Mathematics, 4.

Gaertner, E. (2006). Basic complex boundary value problems in in the upper half plane (Doctoral dissertation).

Tovmasyan N. and Babayan A. (2007). Boundary value problems for second-order elliptic equations in a half-space in the class of functions of polynomial growth (in Russian), Nonclassical equations of mathematical physics, Novosibirsk, 2007, 273-282.

Görmüş, Z. 2008. Analitik Fonksiyonların Yarı Düzlem İçin Riemann Sinır Değer Problemi. Yüksek Lisans Tezi, Kafkas Üniversitesi Fen Bilimleri Enstitüsü Matematik Anabilim Dalı, 28, Kars.

Gürlebeck, K., and Zhang, Z. (2010). Some Riemann boundary value problems in Clifford analysis. Mathematical methods in the applied sciences, 33(3), 287-302.

Abreu-Blaya, R., Bory-Reyes, J., and Kats, B. A. (2011). Integration over non-rectifiable curves and Riemann boundary value problems. Journal of Mathematical Analysis and Applications, 380(1), 177-187.

Sheng-yong, Y. A. N. (2011). Non-normal inverse Riemann-Hilbert boundary value problems for analytic functions [J]. Journal of Lanzhou University of Technology, 2.

Gakhov, F. D. (2014). Boundary value problems. Elsevier.

Aghekyan, S. A. (2016). On a Hilbert problem in the half-plane in the class of continuous functions. Ученые записки Ереванского государственного университета, серия Физические и Математические науки, (2), 9-14.

Vasilyev, V. (2018). Discrete pseudo-differential operators and boundary value problems in a halfspace and a cone. Lobachevskii Journal of Mathematics, 39(2), 289-296.

Hayrapetyan, H. M. (2018, April). On a boundary value problem with infinite index. In International Scientific Conference (on) Modern Methods, Problems and Applications of Operator Theory and Harmonic Analysis (pp. 387-397). Springer, Cham.

Galybin, A. N. (2018). Boundary value problems for elastic half-planes posed in terms of stress and displacement orientations. Boundary Elements and Other Mesh Reduction Methods, 217.

Hu, B. B., Xia, T. C., and Ma, W. X. (2018). Riemann-Hilbert approach for an initial-boundary value problem of the two-component modified Korteweg-de Vries equation on the halfline. Applied Mathematics and Computation, 332, 148-159.

Hayrapetyan, H. M., and Aghekyan, S. A. (2019a). On a Riemann boundary value problem for weighted spaces in the half-plane. Armenian Journal of Mathematics, 11(7), 1-14.

Hayrapetyan, H. M., and Aghekyan, S. A. (2019b). On a Riemann Boundary Value Problem in the Half-plane in the Class of Weighted Continuous Functions. Journal of Contemporary Mathematical Analysis (Armenian Academy of Sciences), 54(2), 79-89.

Bikchantaev, I. A. (2020). Periodic Boundary Value Problem for a Linear Elliptic Equation of the Second Order in a Half-Plane. Differential Equations, 56(7), 813-818.

77 I P a g e

www.iiste.org 
International Journal of Scientific and Technological Research

www.iiste.org

ISSN 2422-8702 (Online), DOI: 10.7176/JSTR/7-01-06

Vol.7, No.1, 2021

IISE

78 | P a g e

www.iiste.org 